\title{
Homogenisation problems in reactive decontamination
}

\author{
E. LUCKINS ${ }^{1}$, C. J. W. BREWARD ${ }^{1}$, I. M. GRIFFITHS ${ }^{1}$, and Z. WILMOTT ${ }^{1}$ \\ ${ }^{1}$ Mathematical Institute, University of Oxford, Radcliffe Observatory Quarter, Oxford, OX2 6GG, UK \\ email: breward@maths.ox.ac.uk
}

(Received 21 June 2020)

\begin{abstract}
The decontamination of hazardous chemical agents from porous media is an important and critical part of the clean-up operation following a chemical-weapon attack. Decontamination is often achieved through the application of a cleanser, which reacts on contact with an agent to neutralise it. While it is relatively straightforward to write down a model that describes the interplay of the agent and cleanser on the scale of the pores in the porous medium, it is computationally expensive to solve such a model over realistic spill sizes.

In this paper, we consider the homogenisation of a porescale model for the interplay between agent and cleanser, with the aim of generating simplified models that can be solved more easily on the spill scale but accurately capture the microscale structure and chemical activity. We consider two situations: one in which the agent completely fills local porespaces and one in which it does not. In the case when the agent does not completely fill the porespace, we use established homogenisation techniques to systematically derive a reaction-diffusion model for the macroscale concentration of cleanser. However, in the case where the agent completely fills the porespace, the homogenisation procedure is more in-depth and involves a two-timescale approach coupled with a spatial boundary layer. The resulting homogenised model closely resembles the microscale model with the effect of the porous material being incorporated into the parameters. The two models cater for two different spill scenarios, and provide the foundation for further study of reactive decontamination.
\end{abstract}

Key Words: Homogenization; Equations in media with periodic structure; Diffusion and convection; Reaction effects in flows; Interface problems.

2010 Mathematics Subject Classification: 35B27 (Primary); 76Rxx (Secondary)

\section{Introduction}

During a liquid-chemical-weapon attack in an urban environment, the chemical agent often lands on, and seeps into, porous building materials such as concrete or tarmac. In order to decontaminate the material, any agent present on the surface is removed, and then a cleaning solution (the "cleanser") is applied to the top of the porous substrate (often using a mop), which reacts with the agent trapped within the pore structure. Often 
the agent and the cleanser are immiscible fluids (typically one is oily and one is aqueous), which means that the decontamination reaction occurs at the interface between the two fluids. It is crucial for public safety that all the agent is reacted away, but it is difficult to tell from surface measurements whether or not any agent remains unreacted inside the material. It is also difficult to predict the time it takes for all the agent to be reacted away: while reaction times may (or may not) be known for the cleanser-agent pair in the laboratory, it is unclear how the porous material and initial three-dimensional shape of the spill affects the process.

The problem of reactive decontamination within a porous medium has been previously studied in [3] and [6]. In [3], flow of a chemical agent into a porous medium is considered; the 2-dimensional analogue is studied in 10, 1 After a discussion of the flow of agent into the porous material, [3] and [6] consider the reactive decontamination process in which the cleanser is assumed to be in aqueous solution and the agent is assumed to be oil based, and they study the diffusion of cleanser and the (harmless) product of the reaction through the aqueous solution, and the chemical reaction at the interface. They derive appropriate interface conditions by conservation of chemical arguments. The decontamination times are found in two cases: (i) when the limiting step is the diffusion of product away from the reaction front, and (ii) when the limiting step is diffusion of cleanser to the front. The key result is that, in certain parameter regimes, increasing the cleanser concentration does not decrease the overall time to decontaminate all the agent. However, the models in [6] do not explicitly take into account the porous structure in which the process occurs.

Given that a typical chemical spill can be tens of centimetres across but the pores of the material are typically of micron size, it is unfeasible to keep explicit track of the local concentration of cleanser everywhere within the porous structure. There is a clear need to build simple models that hold over the whole of the spill, which take into account the effect of the fine-scale structure of the porous medium and the localisation of the decontamination front to the cleanser-agent interface. We will use homogenisation theory to derive "averaged" equations, valid over the entire spill but which take the porescale structure and chemistry into account. Homogenisation techniques have been used very successfully for numerous diffusion-based problems. For instance, [4 and $[5$ consider the diffusion (and advection) of particles suspended in solution through a filter and they allow the porosity of the filter to change over the macroscale, while in 1$]$ the diffusion through a medium with varying porosity is studied and the effective macroscale diffusion coefficient computed, for both periodic and random microstructures. Similar techniques have been used to study the growth of biofilms within a saturated porous medium. For example, Schultz et al. 14, 15 use homogenisation to derive macroscale models for the growth of biofilms and the fluid flow through the medium. The incorporation of chemical reactions into the body of the fluid is studied in [7], where corrosion of the microstructure occurs. They homogenise appropriate microscale models and determine effective reaction and transport coefficients.

Homogenisation methods may also be used to derive macroscale boundary conditions.

1 More generally, the motion of fluids through porous media, often known as "gravity-driven flows", are well-studied problems, with notable papers including 9 and 12 . 

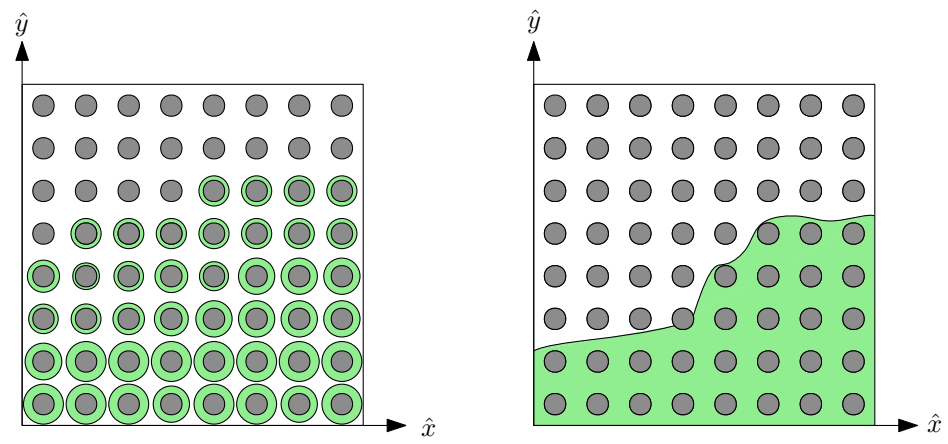

Figure 1. Schematic of the two possible scenarios that we will consider. On the left the agent (green) coats the pore walls, while on the right the agent fills the porespace in some region of the domain. We refer to these as the "unsaturated" and "saturated" models respectively. The cleanser solution (white) fills the remaining porespace.

For instance, the Beavers and Joseph boundary condition at a (static) boundary of a porous medium can be derived via homogenisation (see, for example, 13]). However, the homogenisation of processes happening at a non-static, fluid-fluid interface is a less standard problem.

The progression of the decontamination reaction through the porous substrate depends fundamentally on how the agent is distributed in the medium. For example, it might coat the walls of the pores, or it might completely fill the porespace, as shown in figure 1. We expect that the resulting macroscale model will be different in each of these two canonical scenarios.

In this paper, we will build microscale mathematical models for these two scenarios, which we will homogenise to generate macroscale models that will be applicable on the spill scale. We will then compare and contrast the behaviour of these models in a simple scenario. In more detail, in Section 2 we will build the model that holds within the fluidfilled pores of the substrate. In Section 3, we will perform a homogenisation of these equations, looking at the case in which the chemical agent coats the solid structure, while in Section 4 we will perform the homogenisation in the case where the agent fills the pores. In Section 5 we will compare the two models qualitatively. Concluding remarks are given in Section 6 .

\section{Mathematical model}

In this section we present a model for the decontamination process. We assume that the agent is a neat oily chemical, the cleaning fluid consists of an aqueous solution of the cleanser chemical, and that the two fluids are immiscible so that the decontamination reaction only takes place at the interface between the two fluids. We further assume that the product(s) of the decontamination reaction are only soluble in the aqueous phase so that the agent phase remains undiluted and is depleted during the reaction. We focus on decontamination situations in which the agent has already soaked into a porous substrate and assume that the transport of cleanser is purely due to diffusion within the aqueous solution, so that there is no fluid flow of either agent or cleanser. 
We consider a $2 \mathrm{D}$ porous medium, with $\hat{x}$ and $\hat{y}$ the horizontal and vertical coordinates respectively, as shown in figure 1. Here and henceforth we use hat-notation $\left({ }^{\wedge}\right)$ to signify dimensional variables. We denote the solid structure by the region $\hat{\Omega}_{s}$, the solid-fluid boundary by $\partial \hat{\Omega}_{s}$, and the porespace occupied by fluid by $\hat{\Omega}=\mathbb{R}^{2} \backslash \hat{\Omega}_{s}$. We denote the region of the porespace occupied by cleanser fluid by $\hat{\Omega}_{c} \in \hat{\Omega}$, and that occupied by agent by $\hat{\Omega}_{a}=\hat{\Omega} \backslash \hat{\Omega}_{c}$. The fluid-fluid interface between agent and cleanser is denoted $\partial \hat{\Omega}_{i}$. The concentration of cleanser, $\hat{c}$, measured in $\mathrm{mol} / \mathrm{m}^{3}$, and the volume fraction of agent, $\hat{a}$, therefore satisfy

$$
\begin{array}{lll}
\hat{a}=1, & \hat{c}=0, & \text { in } \hat{\Omega}_{a} \\
\hat{a}=0, & \hat{c}_{\hat{t}}=\hat{D} \hat{\nabla}^{2} \hat{c}, & \text { in } \hat{\Omega}_{c}
\end{array}
$$

where $\hat{D}$ is the spatially uniform diffusivity of the cleanser within the solution. At any interface between the agent and cleanser $\partial \hat{\Omega}_{i}$, the decontamination reaction occurs and conservation of cleanser at this interface reads

$$
+\hat{c} \hat{\boldsymbol{v}} \cdot \boldsymbol{n}+\hat{D} \hat{\nabla} \hat{c} \cdot \boldsymbol{n}=-\hat{j}
$$

where $\hat{\boldsymbol{v}}$ is the velocity of the interface, $\boldsymbol{n}$ is the unit normal to the interface pointing into the chemical agent, and $\hat{j}$ is the flux of cleanser molecules into the reaction. Assuming that no voids are created by the decontamination reaction, conservation of volume reads

$$
-\hat{\boldsymbol{v}} \cdot \boldsymbol{n}=-\hat{\chi} \hat{j}
$$

where $\hat{\chi}$ is the molar volume of agent, measured in $\mathrm{m}^{3} / \mathrm{mol}$. We assume that the chemical reaction between agent molecule $\mathcal{A}$ and cleanser molecule $\mathcal{C}$ is described by

$$
\lambda_{a} \mathcal{A}+\lambda_{c} \mathcal{C} \rightarrow \text { product }
$$

where $\lambda_{a}, \lambda_{c} \in \mathbb{N}$ are the stoichiometry constants. For simplicity, we assume that $\lambda_{a}=$ $\lambda_{c}=1$, although the results could be easily extended to other values. We use the Law of Mass Action to determine the flux $\hat{j}$, and write

$$
\hat{j}=\hat{k} \hat{c},
$$

where $\hat{k}$ is the mass transfer coefficient for the reaction, measured in $\mathrm{m} / \mathrm{s}$. Finally, we assume that there is no flux of cleanser molecules into the solid, and so we write

$$
\hat{\nabla} \hat{c} \cdot \boldsymbol{n}_{s}=0 \quad \text { on } \partial \hat{\Omega}_{s},
$$

where $\boldsymbol{n}_{s}$ is the normal to the solid. In summary, our model reads

$$
\begin{aligned}
& \hat{c}_{\hat{t}}=\hat{D} \hat{\nabla}^{2} \hat{c} \quad \text { in } \quad \hat{\Omega}_{c}, \\
& -\hat{c} \hat{\boldsymbol{v}} \cdot \boldsymbol{n}+\hat{D} \hat{\nabla} \hat{c} \cdot \boldsymbol{n}=-\hat{k} \hat{c} \quad \text { on } \quad \partial \hat{\Omega}_{i}, \\
& -\hat{\boldsymbol{v}} \cdot \boldsymbol{n}=-\hat{\chi} \hat{k} \hat{c} \quad \text { on } \quad \partial \hat{\Omega}_{i}, \\
& \hat{\nabla} \hat{c} \cdot \boldsymbol{n}_{s}=0 \quad \text { on } \quad \partial \hat{\Omega}_{s} \text {, }
\end{aligned}
$$

which must be coupled with appropriate boundary conditions on $\hat{c}$ (e.g., at the top of the porous medium), and an initial condition. This model holds within the complicated 

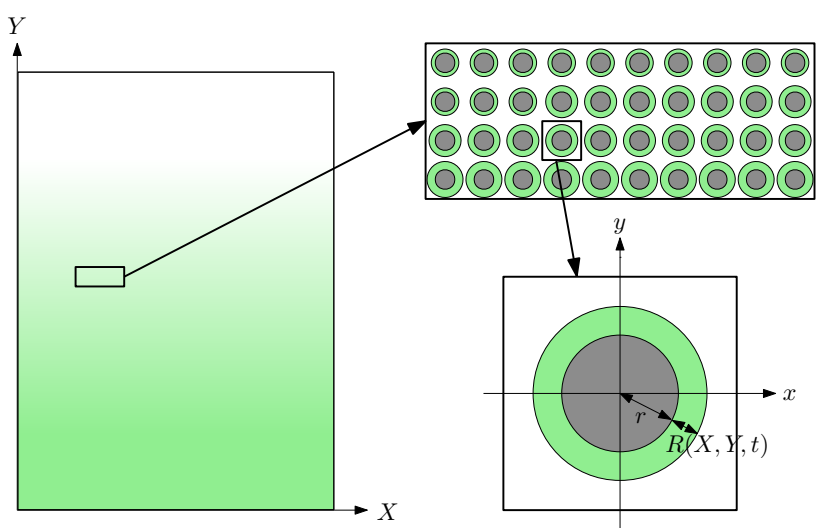

Figure 2. Schematic of the porous structure, and the layer of agent coating the solid in dimensionless variables, introduced in 3.2$) ;(x, y)$ are microscale variables, while $(X, Y)$ are macroscale variables defined in (3.7).

domain $\hat{\Omega}$ around the solid structure of the porous medium. We wish to homogenise this model, and derive averaged equations that hold over the whole domain and take into account the solid microstructure.

Before proceeding, we note that the ratio of the pore lengthscale, $\hat{d}$, to the spill lengthscale, $\hat{L}$, is small, $\epsilon=\hat{d} / \hat{L} \ll 1$, which is necessary for our homogenisation analysis. As is standard in multiple-scales analyses, we will make the key assumption that the dependent variables (eg $\hat{c}$ ) can vary on both the porescale and on the spillscale independently, and we will relate our spatial variables on the macroscale to the microscale using $\epsilon$. We will zoom in to a single "cell" on the microscale, and analyse the behaviour at this level, before averaging to obtain equations that hold on the macroscale, with the explicit microscale dependence averaged out. As described in [8], the macroscale model derived by this method holds in the limit as $\epsilon \rightarrow 0$, and so is a very good approximation for situations in which $\epsilon$ is very small.

In the next two sections, we will consider two canonical scenarios, one in which the agent coats the solid microstructure and another in which the agent completely fills the porespace.

\section{Agent coats the solid microstructure}

In this section, we will use the model (2.8)-2.11) to describe decontamination in the case where the agent has coated the pores (as shown in figure 2), and we will homogenise the equations to find the leading-order macroscale behaviour. We will follow the argument presented in 4, in which the mathematical problem is similar but the application (adsorption of particles onto the solid structure in a filter) is fundamentally different.

For simplicity, we suppose that the microscale solid structure consists of a periodic square lattice of circular solids, each with constant radius $\hat{r}$ and located in the centre of each cell, size $\hat{d}$. The square array periodicity may be relaxed to allow for a more realistic quasiperiodicity but the methodology is similar [11. We assume that the solid structure is coated by a layer of the agent, thickness $\hat{R}$, which is reacted away by the surrounding 
cleanser. The interface speed $\hat{\boldsymbol{v}}$ is thus given by

$$
\hat{\boldsymbol{v}}=-\hat{R}_{\hat{t}} \boldsymbol{n},
$$

since $\boldsymbol{n}$ points into the agent. We note that, while the local normal to the agent-cleanser interface is in the direction $\boldsymbol{e}_{r}$ (the unit vector in the radial direction from the centre of the solid circle), we will later allow the interface to depend on both the macroscale and microscale variables. A key facet of the model will be to allow $\hat{R}$ to vary over the macroscale, and the derivatives in the definition of the normal will need to be altered to account for the dependence of the interface on both the microscale and macroscale; thus the normal $\boldsymbol{n}$ is not simply $\boldsymbol{e}_{r}$.

\subsection{Dimensionless model}

We choose to nondimensionalise the model 2.8 -2.11 using the pore lengthscale (since our equations hold within the fluid region of a pore) but using the timescale associated with diffusion over the spill scale (since we are interested in overall clean-up), and thus we choose

$$
(\hat{x}, \hat{y}, \hat{R})=\hat{d}(x, y, R), \quad \hat{c}=c^{*} c, \quad \hat{t}=\frac{\hat{d}^{2}}{\epsilon^{2} \hat{D}} t,
$$

where $c^{*}$ is the initial concentration of cleanser in the cleaning solution and subscripts denote differentiation. The dimensionless model in the cleanser fluid reads:

$$
\epsilon^{2} c_{t}=\nabla^{2} c
$$

while, at the agent-cleanser interface located at $|\boldsymbol{x}|=r+R$, we have

$$
\epsilon^{2} c R_{t}-\nabla c \cdot \boldsymbol{n}=\epsilon^{2} \beta c, \quad \epsilon^{2} R_{t}=-\epsilon^{2} \beta \gamma c,
$$

when $R>0$ and

$$
\nabla c \cdot \boldsymbol{e}_{r}=0
$$

when $R=0$, where $\boldsymbol{n}$ is the dimensionless normal to the interface. We have introduced three dimensionless parameters:

$$
\beta=\frac{\hat{d} \hat{k}}{\epsilon^{2} \hat{D}}, \quad \gamma=\hat{\chi} c^{*}, \quad r=\frac{\hat{r}}{\hat{d}} .
$$

which represent the ratio of the diffusion timescale over the macroscale to the chemical reaction timescale on the microscale, the amount the interface moves due to these effects, and the dimensionless microscale solid structure radius, respectively. We assume that both $\beta$ and $\gamma$ are order-one constants, so that the resulting model corresponds to the distinguished limit in which the diffusion and reaction terms are both present. This regime also caters for situations in which $\beta$ and $\gamma$ are smaller than $O(1)$. Another asymptotic limit occurs when $\beta \sim \epsilon^{-2}$, in which all the action takes place on the microscale, but we do not consider this here. 


\subsection{Homogenisation}

We now homogenise (3.3)-3.5 to find the macroscale behaviour. We assume that $c$ varies over both the microscale and macroscale and that $R$ varies over the macroscale. We introduce the macroscale variables

$$
X=\epsilon x, \quad Y=\epsilon y,
$$

and thus write $c=c(x, y, X, Y, t)$ and $R=R(X, Y, t)$. We expand the spatial derivatives using the chain rule to account for both sets of variables and replace $\nabla$ with $\nabla_{x}+\epsilon \nabla_{X}$. Since we assume that the microscale solid structure is periodic, we consider a canonical microscale problem that consists of a solid particle coated in agent and surrounded by cleanser solution, as shown in the insert of figure 2, This is called the "unit cell" for this problem, which we denote by $\omega=[0,1]^{2}$. We impose that $c$ is locally periodic in the microscale variables by enforcing periodicity over $\omega$. Since we assume that the microscale and macroscale variables are independent, this periodicity condition does not affect the variation of the cleanser concentration over the macroscale. Further, since $R=R(X, Y, t)$, the part of the cell occupied by cleanser, which we call $\omega_{c}(X, Y, t)$, is also a function of $X, Y$ and $t$.

Substituting this change of variables into (3.3)-3.5), and taking care to remember that the normal to the interface $|\boldsymbol{x}|=r+R$ is

$$
\boldsymbol{n}=\frac{\nabla(r+R-|\boldsymbol{x}|)}{|\nabla(r+R-|\boldsymbol{x}|)|}=\frac{\left(\nabla_{x}+\epsilon \nabla_{X}\right)(r+R-|\boldsymbol{x}|)}{\left|\left(\nabla_{x}+\epsilon \nabla_{X}\right)(r+R-|\boldsymbol{x}|)\right|},
$$

in this revised coordinate system, the model becomes

$$
\epsilon^{2} c_{t}=\nabla_{x}^{2} c+\epsilon\left(\nabla_{x} \cdot \nabla_{X}+\nabla_{X} \cdot \nabla_{x}\right) c+\epsilon^{2} \nabla_{X}^{2} c
$$

in $\omega_{c} \times \mathbb{R}^{2}$, with boundary conditions on $|\boldsymbol{x}|=r+R$, correct to $O\left(\epsilon^{2}\right)$, reading

$$
\epsilon^{2}\left(c R_{t}-\beta c\right)=\left(\nabla_{x}+\epsilon \nabla_{X}\right) c \cdot\left(\nabla_{x}+\epsilon \nabla_{X}\right)(r+R-|\boldsymbol{x}|),
$$

$$
\epsilon^{2} R_{t}=-\epsilon^{2} \beta \gamma c
$$

when $R>0$ and

$$
\left(\nabla_{x}+\epsilon \nabla_{X}\right) c \cdot \boldsymbol{e}_{r}=0,
$$

when $R=0$. We seek asymptotic expansion solutions of $3.9-3.12$ of the form

$$
\begin{aligned}
c & =c_{0}(x, y, X, Y, t)+\epsilon c_{1}(x, y, X, Y, t)+\epsilon^{2} c_{2}(x, y, X, Y, t)+\cdots, \\
R & =R_{0}(X, Y, t)+\epsilon R_{1}(X, Y, t)+\cdots,
\end{aligned}
$$

and we note that we apply the boundary conditions $3.10-(3.12)$ on $\partial \omega_{i 0}=\{|\boldsymbol{x}|=$ $\left.r+R_{0}\right\}$ rather than $\partial \omega_{i}=\{|\boldsymbol{x}|=r+R\}$. This requires us to Taylor expand

$$
\begin{aligned}
\left.f\right|_{|\boldsymbol{x}|=r+R}= & \left.f\right|_{|\boldsymbol{x}|=r+R_{0}}-\left.\left(\epsilon R_{1}+\epsilon^{2} R_{2}+\cdots\right) \boldsymbol{e}_{r} \cdot \nabla_{x} f\right|_{|\boldsymbol{x}|=r+R_{0}} \\
& +\left.\frac{1}{2}\left(\left(\epsilon R_{1}+\epsilon^{2} R_{2}+\cdots\right) \boldsymbol{e}_{r} \cdot \nabla_{x}\right)^{2} f\right|_{|\boldsymbol{x}|=r+R_{0}}+\cdots
\end{aligned}
$$

for any function $f$ depending on $x, y$ in the interface conditions $3.10-3.11$. We denote 
the cleanser-occupied region of a cell outside of $\partial \omega_{i 0}$ by $\omega_{0}(X, Y, t)$ (instead of the region $\omega_{c}$ outside of $\left.\partial \omega_{i}\right)$.

We find that the leading-order problem in $\omega_{0}(X, Y, t)$ is

$$
\nabla_{x}^{2} c_{0}=0,
$$

with, on $\partial \omega_{i 0}$

$$
-\boldsymbol{e}_{r} \cdot \nabla_{x} c_{0}=0,
$$

coupled with periodic boundary conditions on the edge of the unit cell. We note that, at this order, the interface condition 3.10 has reduced to the condition of no flux (3.18) and so the same condition holds whether $R_{0}=0$ or $R_{0}>0$.

We multiply (3.17) by $c_{0}$ and integrate over $\omega_{0}(X, Y, t)$ to find that

$$
0=\iint_{\omega_{0}} c_{0} \nabla_{x}^{2} c_{0} \mathrm{~d} x \mathrm{~d} y=-\iint_{\omega_{0}}\left|\nabla_{x} c_{0}\right|^{2} \mathrm{~d} x \mathrm{~d} y+\int_{\partial \omega_{0}} c_{0} \nabla_{x} c_{0} \cdot \boldsymbol{n} \mathrm{d} s,
$$

where the boundary $\partial \omega_{0}(X, Y, t)$ is the union of the edge of the cell $\partial \omega$ and the interface/solid boundary. By periodicity of $c_{0}$, the line integral around the edge of the cell evaluates to zero, and using the no-flux interface condition the line integral along $\partial \omega_{i 0}$ is also zero. Hence for all times $t$,

$$
0=-\iint_{\omega_{0}(X, Y, t)}\left|\nabla_{x} c_{0}\right|^{2} \mathrm{~d} x \mathrm{~d} y
$$

and we thus conclude that $c_{0}=c_{0}(X, Y, t)$ is independent of the microscale. To close the model for $c_{0}$, we must proceed to higher order in $\epsilon$. The $\mathcal{O}(\epsilon)$ problem in $\omega_{0}(X, Y, t)$ reads

$$
\nabla_{x}^{2} c_{1}=0
$$

with, on $\partial \omega_{i 0}$,

$$
-\boldsymbol{e}_{r} \cdot\left(\nabla_{x} c_{1}+\nabla_{X} c_{0}\right)=0
$$

again coupled with periodic conditions for $c_{1}$ on the boundary of the cell. The linearity of the $\mathcal{O}(\epsilon)$ problem means that we can construct a solution of the form $c_{1}=\boldsymbol{w} \cdot \nabla_{X} c_{0}$, where we choose the components of $\boldsymbol{w}=\left(w_{1}(x, y, X, Y, t), w_{2}(x, y, X, Y, t)\right)$ to solve the cell problems

$$
\begin{aligned}
\nabla_{x}^{2} w_{i}=0 & & \text { in } \omega_{0}(X, Y, t), \\
-\boldsymbol{e}_{r} \cdot\left(\nabla_{x} w_{i}+\boldsymbol{e}_{i}\right)=0 & & \text { on } \partial \omega_{i 0},
\end{aligned}
$$

with $w_{i}$ periodic over the unit cell, for $i=1,2$ where $\boldsymbol{e}_{1}, \boldsymbol{e}_{2}$ are the unit vectors in the $x$ and $y$ directions, respectively. Thus, we can determine $c_{1}$ in terms of $c_{0}$ and the solution of the cell problem, which we note depends on $R_{0}(X, Y, t)$.

The $\mathcal{O}\left(\epsilon^{2}\right)$ problem is more complicated. The governing equation, to be solved in $\omega_{0}(X, Y, t)$, reads

$$
c_{0 t}=\nabla_{x}^{2} c_{2}+\left(\nabla_{x} \cdot \nabla_{X}+\nabla_{X} \cdot \nabla_{x}\right) c_{1}+\nabla_{X}^{2} c_{0},
$$

with, when $R_{0}>0$, the boundary conditions

$$
c_{0} R_{0 t}-\beta c_{0}=\nabla_{X} R_{0} \cdot\left(\nabla_{X} c_{0}+\nabla_{x} c_{1}\right)-\boldsymbol{e}_{r} \cdot\left(\nabla_{x} c_{2}+\nabla_{X} c_{1}\right),
$$




$$
R_{0 t}=-\beta \gamma c_{0},
$$

on $\partial \omega_{i 0}$ while, if $R_{0}=0$, we have the boundary condition

$$
\left(\nabla_{x} c_{2}+\nabla_{X} c_{1}\right) \cdot \boldsymbol{e}_{r}=0
$$

with $c_{2}$ periodic over the unit cell in both cases. We note that we have used the $\mathcal{O}(\epsilon)$ boundary condition 3.22 to simplify 3.26 . We integrate 3.25 over $\omega_{0}(X, Y, t)$, and, using the fact that $c_{0}$ is independent of $x$ and $y$ along with the divergence theorem, obtain

$$
\mathcal{V}\left(R_{0}\right) c_{0 t}=\iint_{\omega_{0}} \nabla_{X} \cdot\left(\nabla_{X} c_{0}+\nabla_{x} c_{1}\right) \mathrm{d} x \mathrm{~d} y+\int_{\partial \omega_{0}}\left(\nabla_{x} c_{2}+\nabla_{X} c_{1}\right) \cdot \boldsymbol{n} \mathrm{d} s,
$$

where $\mathcal{V}\left(R_{0}\right)$ is the area of the cleanser-occupied region, given by

$$
\mathcal{V}\left(R_{0}\right)=\mathcal{V}(X, Y, t):=\iint_{\omega_{0}(X, Y, t)} \mathrm{d} x \mathrm{~d} y=1-\pi\left(r+R_{0}\right)^{2}
$$

in our circular geometry. We first look at the situation where $R_{0}>0$. The area integral in 3.29 may be rewritten using a transport theorem, namely:

$$
\nabla_{X} \cdot \iint_{\omega_{0}(X, Y, t)} \boldsymbol{f}(x, y, X, Y, t) \mathrm{d} x \mathrm{~d} y=\iint_{\omega_{0}} \nabla_{X} \cdot \boldsymbol{f} \mathrm{d} x \mathrm{~d} y-\int_{\partial \omega_{i 0}} \nabla_{X} R_{0} \cdot \boldsymbol{f} \mathrm{d} s
$$

for any function $\boldsymbol{f}$. The line integral in 3.29 may be split into two parts: the edge of the cell $\partial \omega$, and the fluid-fluid interface $\partial \omega_{i 0}$. The integral around the edge of the cell evaluates to zero because of the periodicity of $c_{1}$ and $c_{2}$, while the integral over the fluid-fluid interface is

$$
\begin{aligned}
\int_{\partial \omega_{i 0}}\left(\nabla_{x} c_{2}\right. & \left.+\nabla_{X} c_{1}\right) \cdot \boldsymbol{n}_{x} \mathrm{~d} s=\int_{\partial \omega_{i 0}}-\beta c_{0}+c_{0} R_{0 t}-\nabla_{X} R_{0} \cdot\left(\nabla_{X} c_{0}+\nabla_{x} c_{1}\right) \mathrm{d} s \\
& =-\mathcal{L}\left(R_{0}\right)\left(\beta c_{0}+\beta \gamma c_{0}^{2}\right)-\int_{\partial \omega_{i 0}} \nabla_{X} R_{0} \cdot\left(\nabla_{X} c_{0}+\nabla_{x} c_{1}\right) \mathrm{d} s,
\end{aligned}
$$

where we have used (3.26), (3.27), and the fact that $c_{0}$ is independent of $x$ and $y$. We have also introduced the local length $\mathcal{L}\left(R_{0}\right)$ of the agent-cleanser interface $\partial \omega_{i 0}$, defined by

$$
\mathcal{L}\left(R_{0}\right):=\int_{\partial \omega_{i 0}} \mathrm{~d} s=2 \pi\left(r+R_{0}\right) .
$$

in our circularly symmetric geometry. We use (3.31 to manipulate the double integral in 3.29 and substitute 3.32 and $c_{1}=\boldsymbol{w} \cdot \nabla_{X} c_{0}$ to find that 3.29 reduces to the reaction-diffusion equation

$$
\mathcal{V}\left(R_{0}\right) c_{0 t}=\nabla_{X} \cdot\left(\underline{\underline{D}}\left(R_{0}\right) \mathcal{V}\left(R_{0}\right) \nabla_{X} c_{0}\right)-\mathcal{L}\left(R_{0}\right)\left(1+\gamma c_{0}\right) \beta c_{0}
$$

where the entries in the diffusivity tensor $\underline{\underline{D}}$ are given by

$$
D_{i j}=\delta_{i j}+\frac{1}{\mathcal{V}\left(R_{0}\right)} \iint_{\omega_{0}(X, Y, t)} \frac{\partial w_{j}}{\partial x_{i}} \mathrm{~d} x \mathrm{~d} y .
$$

We note that $\underline{\underline{D}}$ depends on $R_{0}$ via the integral over $\omega_{0}\left(R_{0}\right)$ and that, due to the porescale symmetry of the problem, $\underline{\underline{D}}$ is proportional to the identity matrix. Had we assumed 
a different pore geometry, we would have obtained the same form for (3.34), but with different formulae for $\underline{\underline{D}}, \mathcal{V}$ and $\mathcal{L}$.

Finally, we study the situation with $R_{0}=0$. The analysis is simpler than in the $R_{0}>0$ case and, instead of (3.26) and (3.27), we substitute (3.28) into (3.29) and the line integral immediately evaluates to zero. Since the cell domain $\omega_{0}$ no longer depends on $X$ or $Y$, the area integral in (3.29) may be re-written by moving the derivatives to the outside of the integral, and we find that

$$
c_{0 t}=\nabla_{X} \cdot\left(\underline{\underline{D}}^{0} \nabla_{X} c_{0}\right),
$$

where $\underline{\underline{D}}^{0}$ is given by $(3.35)$ evaluated at $R_{0}=0$.

\subsection{Summary}

In summary, we have derived a macroscopic model for the leading-order cleanser concentration $c:=c_{0}(X, Y, t)$ and the leading-order thickness of the agent layer $R:=R_{0}(X, Y, t)$, which depend only on macroscale variables $X$ and $Y$, but incorporate the microscale effects. Our model reads

$$
c_{t}=\frac{1}{\mathcal{V}(R)} \nabla_{X} \cdot\left(\underline{\underline{D}} \mathcal{V}(R) \nabla_{X} c\right)-\mathcal{F}(R)(1+\gamma c) \beta c, \quad R_{t}=-\beta \gamma c,
$$

when $R>0$, where $\mathcal{F}(R)=\mathcal{L}(R) / \mathcal{V}(R)$, and reduces to

$$
c_{t}=\nabla_{X} \cdot\left(\underline{\underline{D}}^{0} \nabla_{X} c\right),
$$

when $R=0$. Our model must be solved with appropriate (macroscopic) boundary conditions for $c$ and initial conditions for $c$ and $R$. These will depend on the decontamination scenario under consideration.

\section{Agent saturates the porespace; sharp interface on the macroscale}

In this section, we consider the situation where the agent saturates the porous medium in some regions and the cleanser solution saturates the porous media in other regions, and there is a sharp interface between the regions, as shown in figure 1 (right). We assume that the porous medium has the same microscale structure as introduced in section 3 . but instead of tracking the thickness, $\hat{R}$, of the layer of the layer of agent around each inclusion, we track the position of the microscale interface between the two fluids, which we assume to be located at $\hat{y}=\hat{h}(\hat{x}, \hat{t})$, with cleanser diffusing in $\hat{y}>\hat{h}$ and neat agent in $\hat{y}<\hat{h}$.

\subsection{Dimensionless model}

We choose to nondimensionalise the model 2.8 - 2.11) using the pore lengthscale and an intermediate timescale lying between that for diffusion over the pore scale and diffusion over the spill scale,

$$
(\hat{x}, \hat{y}, \hat{h})=\hat{d}(x, y, h), \quad \hat{c}=c^{*} c, \quad \hat{t}=\frac{\hat{d}^{2}}{\epsilon \hat{D}} t .
$$


The timescale, $\hat{d}^{2} / \epsilon \hat{D}$, is chosen to match the movement of the microscale interface due to the chemical reaction. The other process present in our model is diffusion, which, on the pore scale occurs on the much shorter timescale $\hat{d}^{2} / \hat{D}$. Unlike in Section 3 we do not need to specify the shape of the porous microstructure and only require that it is locally periodic. The choice of circular microstructure in figures 1 and 3 is for illustration only.

The dimensionless model reads

$$
\epsilon c_{t}=\nabla^{2} c
$$

in the region of space occupied by cleanser $\left(\Omega_{h}=\Omega \cap\{y>h(x, t)\}\right)$ with, on the agentcleanser interface $y=h(x, t)$,

$$
-\epsilon c \frac{h_{t}}{\sqrt{1+h_{x}^{2}}}+\boldsymbol{n}_{f} \cdot \nabla c=-\epsilon \beta^{*} c,
$$

where $\boldsymbol{n}_{f}$ is the dimensionless downward pointing normal to the interface which can be written as

$$
\boldsymbol{n}_{f}=\frac{1}{\sqrt{1+h_{x}^{2}}}\left(\begin{array}{c}
h_{x} \\
-1
\end{array}\right)
$$

and

$$
\frac{h_{t}}{\sqrt{1+h_{x}^{2}}}=-\gamma \beta^{*} c .
$$

Where the cleanser meets the surface of the substrate, $\partial \Omega_{s} \cap\{y>h(x, t)\}$, we have

$$
\nabla c \cdot \boldsymbol{n}_{s}=0
$$

In this case, we have introduced the dimensionless parameter

$$
\beta^{*}=\frac{\hat{d} \hat{k}}{\epsilon \hat{D}},
$$

and $\gamma=\hat{\chi} c^{*}$ as in Section 3. As in Section 3, $\gamma$ represents the amount the interface moves due to the chemical reaction, but now $\beta^{*}$ is the ratio of the diffusion timescale on the macroscale to the chemical reaction timescale, also on the macroscale (rather than using the chemical reaction timescale on the microscale as we used to define $\beta$ in $(3.6)$ ). This is because, rather than the cleanser-agent interface moving on the microscale due to the chemical reaction as in Section 3 , we are now interested in the chemical-reaction-driven motion of a macroscale boundary. If we assume that $\beta^{*}$ and $\gamma$ are order one, then this macroscale boundary will move at an order-one velocity over the macroscale.

\subsection{Homogenisation}

Our aim is to homogenise the equations and derive the leading-order behaviour of the cleanser concentration and the movement of the macroscale interface over the large lengthscale of the spill. We use the same macroscale spatial variables as used in Section 3 but we also introduce a macroscale time variable $T=\epsilon t$, which is the timescale for diffusion over the macroscale, and (as we shall see later) also the timescale over which the macroscale interface moves. Multiple timescales are necessary for this homogenisation as the timescale $T$ of the macroscale problem is much longer than the timescale $t$ over which 


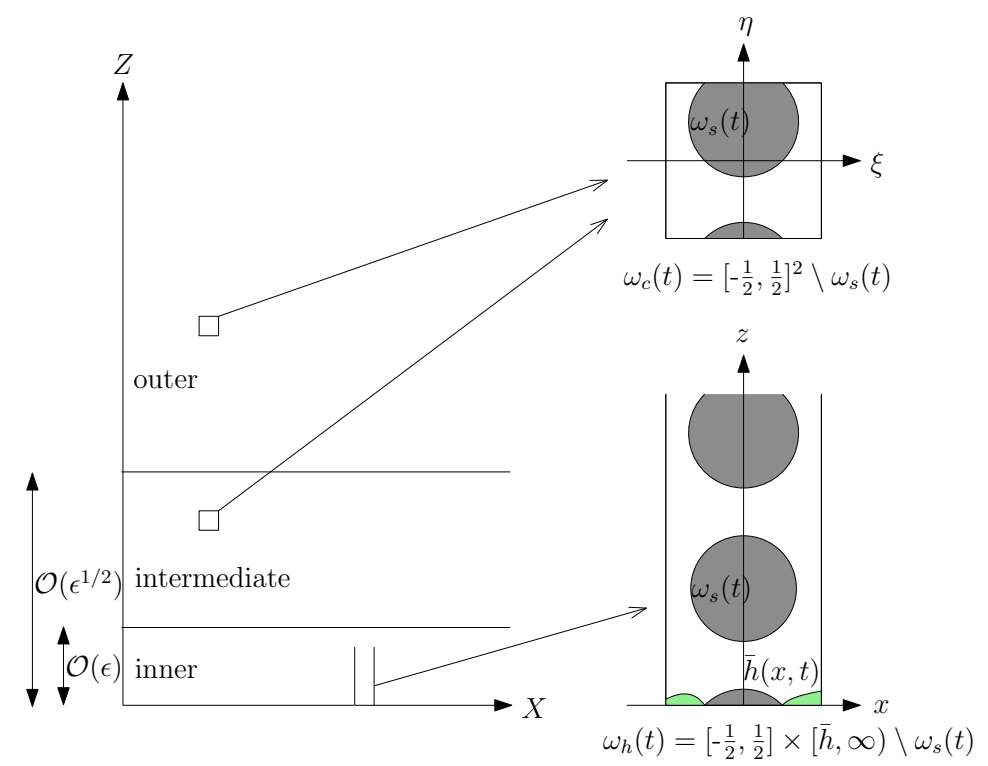

Figure 3. Schematic for the agent-saturated model, with the microscale cells both at $\left(\omega_{h}\right)$ and away from $\left(\omega_{c}\right)$ the interface.

the interface speed oscillates on the microscale (due to the porous structure interfering with the chemical reaction at the interface).

If we consider the problem in terms of the macroscale variables, we see that there are (at least) two different regions in the domain. Firstly, far enough away from the agentcleanser interface, the problem becomes the standard problem of diffusion in a porous medium, which we may homogenise to give an adapted diffusion equation. Secondly, there is the interface region itself. The agent-cleanser interface is necessarily a microscale structure, since the interface only exists in the pores between the solid structure. However we wish to define a boundary for the macroscale problem, moving at an average velocity which does not vary over the timescale of the microscale interface moving through a cell. Due to the fast variation in the interface region, we will consider this as a boundary layer of the macroscale problem. Hence we will refer to the "far-field" region as the outer problem, and the interface region as the inner problem. We will see that the boundarylayer width is order $\epsilon$ in terms of the outer/macroscale variables. In the outer problem, we assume periodicity of the microscale problem over a unit cell, as usual. In the inner problem this clearly cannot hold, and we only assume periodicity across a unit cell in the direction parallel to the macroscale interface, while the microscale variable perpendicular to the interface will be matched to the outer problem.

As we will see, we cannot directly match the inner and outer solutions. This is because, at the interface, the pore lengthscale is too small for the oscillations due to the interface motion to be smoothed out by diffusion. We therefore require an intermediate region between the inner and outer problems, with lengthscale $\epsilon^{1 / 2}$ (which is the diffusion lengthscale over the fast timescale), over which the interface oscillations smooth out to match to the outer problem. A schematic in shown in figure 3 
Specifically, we first consider the problem in terms of macroscale variables $X=\epsilon x$, $Y=\epsilon y$ and $T=\epsilon t$. For simplicity, we assume that the macroscale fluid-fluid interface is flat and is given by $Y=H(T)$; a non-flat macroscale interface would be given by $H=H(X, T)$. The speed of the interface, $v(T):=H_{T}(T)$, will be determined during the homogenisation. Note that $v$ is a function of the slow timescale $T$ only, since we will average over the fast timescale. We change variables to move with this interface, setting $Z:=Y-H(T)$.

Far away from the interface, now located at $Z=0$, we have a standard diffusion in porous media problem, and hence introduce microscale cell variables $\eta=Z / \epsilon, \xi=X / \epsilon$. We must also take into account variation on the fast timescale $t=T / \epsilon$. In this outer region, the concentration $C$ satisfies

$$
\epsilon\left(C_{t}+\epsilon C_{T}-H_{T} C_{\eta}-\epsilon H_{T} C_{Z}\right)=\nabla_{\xi}^{2} C+\epsilon\left(\nabla_{\xi} \cdot e_{2} \frac{\partial}{\partial Z}+e_{2} \frac{\partial}{\partial Z} \cdot \nabla_{\xi}\right) C+\epsilon^{2} C_{Z Z},
$$

when $(\xi, \eta, X, Z) \in \omega_{c}(t) \times \mathbb{R}^{2}$, with

$$
\left(\nabla_{\xi}+\epsilon \boldsymbol{e}_{2} \frac{\partial}{\partial Z}\right) C \cdot \boldsymbol{n}_{s}=0
$$

on the solid surface. Since this coordinate system is moving with the interface, the solid surface is moving up through the microscale cell $\omega_{c}$, with speed $H_{T}(T)$. Here, $\boldsymbol{e}_{2}$ is the unit vector in the $Z$, or $\eta$, direction. We assume that the function $s$, where $s(x, y)=0$ defines the solid boundary $\partial \omega_{s}$, is 1-periodic in both arguments, so that the solid part of the cell is everywhere periodic. The normal to the solid, $\boldsymbol{n}_{s}$ is given by

$$
\boldsymbol{n}_{s}(x, y)=\frac{\nabla_{x} s(x, y)}{\left|\nabla_{x} s(x, y)\right|}=\frac{\nabla_{\xi} s\left(\xi, \eta+H_{T} t\right)}{\left|\nabla_{\xi} s\left(\xi, \eta+H_{T} t\right)\right|},
$$

and is independent of the spatial macroscale variables. We assume that $C$ is periodic in $\xi$ and $\eta$ with period 1 and also in $t$ with period $1 / H_{T}$, which will allow us to average over the microscale.

Near the interface $Z=0$, we have a boundary layer where microscale effects are important. We change variables by defining $z:=Z / \epsilon$, and also introduce the microscale variable $x:=X / \epsilon$. We denote the variation in the interface position due to microscale effects by $z=\bar{h}(x, t)$, which is independent of the macroscale, and periodic in both $x$ (period 1) and $t$ (period $1 / H_{T}$ ), so that the interface speed $H_{T}(T)$ is truly the locally averaged interface speed. Since none of the variables depend on $X$, the only spatial derivatives are $\nabla_{x}=\left(\partial_{x}, \partial_{z}\right)$. The equations in this inner region are therefore

$$
\epsilon\left(c_{t}+\epsilon c_{T}-H_{T} c_{z}\right)=\nabla_{x}^{2} c
$$

in $\omega_{h}(t)$ with, on the microscale boundary $\omega_{h}(t) \cap\{z=\bar{h}\}$

$$
\begin{aligned}
-\epsilon c \frac{\left(H_{T}+\bar{h}_{t}\right)}{\sqrt{1+\bar{h}_{x}^{2}}}+\boldsymbol{n} \cdot \nabla_{x} c & =-\epsilon \beta^{*} c, \\
\frac{\left(H_{T}+\bar{h}_{t}\right)}{\sqrt{1+\bar{h}_{x}^{2}}} & =-\gamma \beta^{*} c,
\end{aligned}
$$


and with, on the solid surface $\partial \omega_{s}(t)$,

$$
\nabla_{x} c \cdot \boldsymbol{n}_{s}=0 .
$$

We further impose that $c$ is periodic in $x$ with period 1 , and in $t$ with period $1 / H_{T}$.

As previously discussed, it transpires that we also require an intermediate layer between the outer and inner problems, with lengthscale chosen to balance diffusion on the $t$-timescale. In this intermediate layer, fast variations from the inner problem are smoothed out by diffusion in order to correctly match with the outer problem. For our intermediate spatial variable, we define $\tilde{z}:=\epsilon^{-1 / 2} Z$, and $\tilde{x}:=\epsilon^{-1 / 2} X$ (although in our case there is no dependence of any variables on $\tilde{x}$, because there is no dependence on $X)$. We again introduce microscale variables $\xi$ and $\eta$ as for the outer problem in order to homogenise over the microscale effects. The cleanser concentration $\tilde{c}$ in this intermediate layer therefore satisfies

$$
\epsilon\left(\tilde{c}_{t}+\epsilon \tilde{c}_{T}-H_{T} \tilde{c}_{\eta}-\epsilon^{1 / 2} H_{T} \tilde{c}_{\tilde{z}}\right)=\nabla_{\xi}^{2} \tilde{c}+\epsilon^{1 / 2}\left(\nabla_{\xi} \cdot \boldsymbol{e}_{2} \frac{\partial}{\partial \tilde{z}}+\boldsymbol{e}_{2} \frac{\partial}{\partial \tilde{z}} \cdot \nabla_{\xi}\right) \tilde{c}+\epsilon \tilde{c}_{\tilde{z} \tilde{z}}
$$

with, on $\partial \omega_{s}$,

$$
\left(\nabla_{\xi}+\epsilon^{1 / 2} \boldsymbol{e}_{2} \frac{\partial}{\partial \tilde{z}}\right) \tilde{c} \cdot \boldsymbol{n}_{s}=0,
$$

and we assume that $\tilde{c}$ is periodic in $\xi$ and $\eta$, with period 1 , and in $t$, with period $1 / H_{T}$.

Since we have $\epsilon^{1 / 2}$ terms in the intermediate region we expand all the dependent variables in powers of $\epsilon^{1 / 2}$ in all regions, in the form

$$
f=f_{0}+\epsilon^{1 / 2} f_{1 / 2}+\epsilon f_{1}+\epsilon^{3 / 2} f_{3 / 2}+\epsilon^{2} f_{2}+\cdots,
$$

remembering that $c=c(x, z, t, T), \bar{h}=\bar{h}(x, t), C=C(\xi, \eta, t, Z, T)$ and $\tilde{c}=\tilde{c}(\xi, \eta, t, \tilde{z}, T)$, with these forms retained at all orders in the expansion. For ease of notation we drop the overbar for $h$. A flowchart showing the information flow through the homogenisation process is shown in figure 4, this may be helpful to refer to through the analysis that follows.

\subsubsection{The outer problem}

We begin by considering the outer problem, given by (4.8)-4.9) along with the periodicity conditions. The leading-order problem reads

$$
\nabla_{\xi}^{2} C_{0}=0
$$

in $\omega_{c}(t)$ with, on $\partial \omega_{s}$,

$$
\nabla_{\xi} C_{0} \cdot \boldsymbol{n}_{s}=0
$$

along with the periodicity of $C_{0}$ over $\omega_{c}$, and in $t$ with period $1 / H_{T}$. Multiplying 4.18 through by $C_{0}$, integrating over $\omega_{c}$, and applying the boundary condition on $\partial \omega_{s}$ and the periodicity at the edge of the cell, we find that $C_{0}=C_{0}(t, Z, T)$ is independent of $\xi$ and $\eta$. Appealing to the linearity of the outer problem and the fact that $\epsilon^{1 / 2}$ does not appear in the outer problem, we obtain the same problem for $C_{1 / 2}$ at $\mathcal{O}\left(\epsilon^{1 / 2}\right)$ as for $C_{0}$ at $\mathcal{O}(1)$, and likewise conclude that $C_{1 / 2}=C_{1 / 2}(t, Z, T)$. 


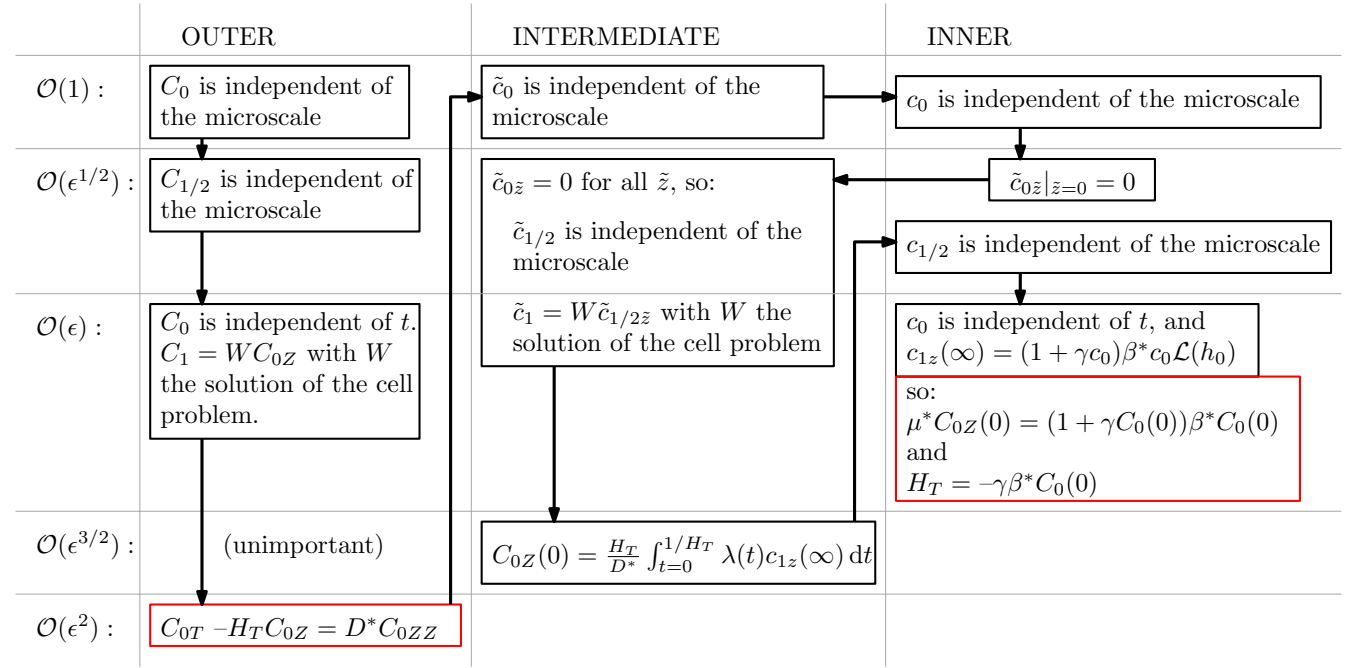

Figure 4. A flow chart displaying the information flow for the sharp-interface homogenisation. The arrows show the direction of flow, and the red boxes highlight the crucial results that form the macroscale model.

At $\mathcal{O}(\epsilon)$, we have

$$
C_{0 t}-H_{T} C_{0 \eta}=\nabla_{\xi}^{2} C_{1}+\left(\nabla_{\xi} \cdot e_{2} \frac{\partial}{\partial Z}+e_{2} \frac{\partial}{\partial Z} \cdot \nabla_{\xi}\right) C_{0},
$$

in $\omega_{c}(t)$, with, on $\partial \omega_{s}$,

$$
\left(\nabla_{\xi} C_{1}+e_{2} C_{0 Z}\right) \cdot \boldsymbol{n}_{s}=0
$$

with $C_{1}$ periodic in $\xi$ and $\eta$ with period 1 , and in $t$ with period $1 / H_{T}$. Since $C_{0}$ is independent of $\xi$ and $\eta$, we integrate 4.20 over $\omega_{c}(t)$ to find that

$$
\mathcal{V} C_{0 t}=\iint_{\omega_{c}(t)} \nabla_{\xi} \cdot\left(\nabla_{\xi} C_{1}+\boldsymbol{e}_{2} C_{0 Z}\right) \mathrm{d} \xi \mathrm{d} \eta=\int_{\partial \omega_{c}(t)}\left(\nabla_{\xi} C_{1}+\boldsymbol{e}_{2} C_{0 Z}\right) \cdot \boldsymbol{n} \mathrm{d} s=0,
$$

where we have used 4.21 on $\partial \omega_{s}$ and the periodicity of $C_{1}$ and $C_{0}$ on the cell boundary, and we introduce the notation

$$
\mathcal{V}=\iint_{\omega_{c}(t)} \mathrm{d} \xi \mathrm{d} \eta
$$

for the cleanser-occupied area of the unit cell. We note that, although the solid is still moving up through the cell at speed $H_{T}$ because of the travelling-wave coordinates, due to the periodicity of the solid structure, the area $\mathcal{V}$ is independent of $t$.

From 4.22 we conclude that $C_{0 t}=0$, i.e., $C_{0}$ is independent of the fast timescale $t$. This simplifies the $\mathcal{O}(\epsilon)$ problem greatly. Appealing to the linearity of this problem and the fact that $C_{0}$ is independent of $\xi$ and $\eta$, we write $C_{1}=W C_{0 Z}$, where $W(\xi, \eta, t)$ is the solution of the cell problem:

$$
\nabla_{\xi}^{2} W=0
$$


in $\omega_{c}(t)$, with, on $\partial \omega_{s}$

$$
\left(\nabla_{\xi} W+\boldsymbol{e}_{2}\right) \cdot \boldsymbol{n}_{s}=0
$$

and with $W$ periodic in $\xi$ and $\eta$ with period 1 , and in $t$, with period $1 / H_{T}$.

By direct analogy with the $\mathcal{O}(\epsilon)$ problem, the $\mathcal{O}\left(\epsilon^{3 / 2}\right)$ equations and boundary conditions give that $\left(C_{1 / 2}\right)_{t}=0$, and that $C_{3 / 2}=W C_{1 / 2}$ with $W$ the solution of 4.24 - 4.25), although we will not need $C_{1 / 2}$ or $C_{3 / 2}$ for the rest of the problem. At $\mathcal{O}\left(\epsilon^{2}\right)$, the problem reads

$$
C_{1 t}-H_{T} C_{1 \eta}+C_{0 T}-H_{T} C_{0 Z}=\nabla_{\xi}^{2} C_{2}+\nabla_{\xi} \cdot e_{2} C_{1 Z}+e_{2} \frac{\partial}{\partial Z} \cdot \nabla_{\xi} C_{1}+C_{0 Z Z},
$$

in $\omega_{c}(t)$ with, on $\partial \omega_{s}$,

$$
\left(\nabla_{\xi} C_{2}+e_{2} C_{1 Z}\right) \cdot \boldsymbol{n}_{s}=0 .
$$

Since the solid $\omega_{s}$ moves through the cell with velocity $-H_{T} \boldsymbol{e}_{2}$, Reynolds Transport Theorem gives that, for any function $F$ which is periodic over the cell,

$$
\begin{aligned}
\frac{\mathrm{d}}{\mathrm{d} t}\left(\iint_{\omega_{c}} F \mathrm{~d} \xi \mathrm{d} \eta\right) & =\iint_{\omega_{c}(t)} F_{t} \mathrm{~d} \xi \mathrm{d} \eta-\int_{\partial \omega_{s}(t)} F H_{T} \boldsymbol{e}_{2} \cdot \boldsymbol{n}_{s} \mathrm{~d} s \\
& =\iint_{\omega_{c}(t)} F_{t}-\nabla_{\xi} \cdot\left(F H_{T} \boldsymbol{e}_{2}\right) \mathrm{d} \xi \mathrm{d} \eta=\iint_{\omega_{c}(t)} F_{t}-H_{T} F_{\eta} \mathrm{d} \xi \mathrm{d} \eta .
\end{aligned}
$$

We integrate (4.26) over $\omega_{c}$ and then over $t$ from 0 to $1 / H_{T}$. Using (4.28), the first terms on the left are

$$
\int_{t=0}^{1 / H_{T}} \iint_{\omega_{c}} C_{1 t}-H_{T} C_{1 \eta} \mathrm{d} \xi \mathrm{d} \eta \mathrm{d} t=\int_{t=0}^{1 / H_{T}} \frac{\mathrm{d}}{\mathrm{d} t}\left(\iint_{\omega_{c}} C_{1} \mathrm{~d} \xi \mathrm{d} \eta\right) \mathrm{d} t=0,
$$

where we have used the periodicity of $C_{1}$ in $t$. Using the divergence theorem, the boundary condition (4.27), the periodicity of $C_{1}$ and $C_{2}$, the facts that $C_{1}=W C_{0 Z}$ and that $C_{0}$ is independent of $t$ and $\xi, \eta$, and the independence of $\omega_{c}$ on $Z$, the remaining terms are

$$
\begin{aligned}
& \frac{\mathcal{V}}{H_{T}}\left(C_{0 T}-H_{T} C_{0 Z}\right) \\
& \quad=\int_{0}^{1 / H_{T}} \iint_{\omega_{c}}\left(\nabla_{\xi} \cdot\left(\nabla_{\xi} C_{2}+e_{2} C_{1 Z}\right)+e_{2} \frac{\partial}{\partial Z} \cdot \nabla_{\xi} C_{1}+C_{0 Z Z}\right) \mathrm{d} \xi \mathrm{d} \eta \mathrm{d} t \\
& \quad=\int_{0}^{1 / H_{T}} \frac{\partial}{\partial Z}\left(\iint_{\omega_{c}}\left(W_{\eta}+1\right) \mathrm{d} \xi \mathrm{d} \eta\right) C_{0 Z} \mathrm{~d} t \\
& \quad=\left(\int_{0}^{1 / H_{T}} \iint_{\omega_{c}}\left(W_{\eta}+1\right) \mathrm{d} \xi \mathrm{d} \eta \mathrm{d} t\right) C_{0 Z Z} .
\end{aligned}
$$

This gives our macroscale convection-diffusion equation for the leading-order cleanser concentration, and can be written as:

$$
C_{0 T}-H_{T} C_{0 Z}=D^{*} C_{0 Z Z},
$$

where

$$
D^{*}:=H_{T} \int_{t=0}^{1 / H_{T}} \lambda(t) \mathrm{d} t, \quad \text { and } \quad \lambda(t):=\frac{1}{\mathcal{V}} \iint_{\omega_{c}}\left(W_{\eta}+1\right) \mathrm{d} \xi \mathrm{d} \eta,
$$

where we are able to take $\mathcal{V}$ through the integral sign because it is independent of $t$. 


\subsubsection{Inner region}

We now consider the inner problem before continuing with the intermediate problem, although we note that the two are intertwined (the flow of information between the regions is shown in figure 4. At leading order in $\epsilon$, the inner problem 4.11 4.14 becomes:

$$
\nabla_{x}^{2} c_{0}=0
$$

in $\omega_{h_{0}}(t):=[-1 / 2,1 / 2] \times\left[h_{0}, \infty\right) \backslash \omega_{s}(t)$ with, on $\partial \omega_{s}$,

$$
\nabla_{x} c_{0} \cdot \boldsymbol{n}_{s}=0,
$$

and, on $z=h_{0}(x, t)$,

$$
\nabla_{x} c_{0} \cdot \boldsymbol{n}_{0}=0
$$

with $c_{0}$ periodic in $x$ with period 1 , and where the vector

$$
\boldsymbol{n}_{0}=\frac{1}{\sqrt{1+h_{0 x}^{2}}}\left(\begin{array}{c}
h_{0 x} \\
1
\end{array}\right)
$$

is normal to the leading-order interface $z=h_{0}(x, t)$. At higher orders, Taylor expanding the boundary conditions about at $z=h_{0}$ introduces new terms which we will highlight as necessary. We also make use of the matching condition

$$
\lim _{z \rightarrow \infty} c_{0}=\lim _{\tilde{z} \rightarrow 0} \tilde{c}_{0},
$$

where, as we will see later, $\tilde{c}_{0}$ is independent of $\xi$ and $\eta$, and hence of $x$ and $z$. Multiplying 4.33 by $c_{0}$ and integrating over the cell $\omega_{h_{0}}(t)$, we have

$$
0=\iint_{\omega_{h_{0}}} c_{0} \nabla_{x}^{2} c_{0} \mathrm{~d} x \mathrm{~d} z=-\iint_{\omega_{h_{0}}}\left|\nabla_{x} c_{0}\right|^{2} \mathrm{~d} x \mathrm{~d} z+\int_{\partial \omega_{h_{0}}} c_{0} \nabla_{x} c_{0} \cdot \boldsymbol{n} \mathrm{d} s .
$$

Applying boundary conditions (4.34) and 4.35), and using the fact that, as $z \rightarrow \infty$, $\nabla_{x} c_{0} \rightarrow 0$, since $\tilde{c}_{0}$ is independent of $x$ and $z$, the boundary integral evaluates to zero and we conclude that $c_{0}$ is independent of $x$ and $z$. At $\mathcal{O}\left(\epsilon^{1 / 2}\right)$, we find that

$$
\nabla_{x}^{2} c_{1 / 2}=0
$$

in $\omega_{h_{0}}(t)$, with, on $\partial \omega_{s}$,

$$
\nabla_{x} c_{1 / 2} \cdot \boldsymbol{n}_{s}=0
$$

and, on $z=h_{0}(x, t)$

$$
\nabla_{x} c_{1 / 2} \cdot \boldsymbol{n}_{0}=0
$$

with $c_{1 / 2}$ periodic in $x$. We note that we have already removed the extra terms in (4.41) that appear from the Taylor expansion about $h_{0}$, since these are all zero. We also have the matching condition

$$
\lim _{\tilde{z} \rightarrow 0} \tilde{c}_{0 \tilde{z}}=\lim _{z \rightarrow \infty} c_{1 / 2 z}
$$

Integrating 4.39 over $\omega_{h_{0}}(t)$, and using the divergence theorem, the boundary conditions 4.40 and 4.41, the periodicity of $c_{1 / 2}$ in $x$, the matching condition 4.42, and the fact 
that $\tilde{c}_{0}$ is independent of $x=\xi$, we find that

$$
0=\iint_{\omega_{h_{0}}} \nabla_{x}^{2} c_{1 / 2} \mathrm{~d} x \mathrm{~d} z=\int_{\partial \omega_{h_{0}}} \nabla_{x} c_{1 / 2} \cdot \boldsymbol{n} \mathrm{d} s=-\left.\int_{x=-\frac{1}{2}}^{\frac{1}{2}} c_{1 / 2 z}\right|_{z \rightarrow \infty} \mathrm{d} x=\left.\tilde{c}_{0 \tilde{z}}\right|_{\tilde{z}=0}
$$

We will use the fact that $\tilde{c}_{0 \tilde{z}}=0$ at $\tilde{z}=0$ later in order to show that $\tilde{c}_{0 \tilde{z}}=0$ for all $\tilde{z}$. To make further progress with the inner problem, we analyse (4.39- 4.41) again. We follow a similar argument as for the $\mathcal{O}(1)$ problem, using the matching condition (see [2]),

$$
\tilde{c}_{1 / 2}(0)=\lim _{z \rightarrow \infty}\left(c_{1 / 2}(z)-z \tilde{c}_{0 \tilde{z}}(0)\right),
$$

along with $\left.\tilde{c}_{0 \tilde{z}}\right|_{\tilde{z}=0}=0$ and the fact that $\tilde{c}_{1 / 2}$ is independent of $\xi$ and $\eta$ (which will be shown later), to conclude that $c_{1 / 2}$ is independent of $x$ and $z$. Finally, we study the $\mathcal{O}(\epsilon)$ problem in the inner region, which reads

$$
c_{0 t}=\nabla_{x}^{2} c_{1}
$$

in $\omega_{h_{0}}(t)$, since $c_{0}$ is independent of $z$, and with, on $z=h_{0}(x, t)$,

$$
\begin{aligned}
-c_{0} \frac{\left(H_{T}+h_{0 t}\right)}{\sqrt{1+h_{0 x}^{2}}}+\boldsymbol{n}_{\mathbf{0}} \cdot \nabla_{x} c_{1}=-\beta^{*} c_{0}, \\
\frac{\left(H_{T}+h_{0 t}\right)}{\sqrt{1+h_{0 x}^{2}}}=-\gamma \beta^{*} c_{0},
\end{aligned}
$$

and on $\partial \omega_{s}$,

$$
\nabla_{x} c_{1} \cdot \boldsymbol{n}_{s}=0
$$

with $c_{1}$ periodic in $x$ and $t$. Further, since $\tilde{c}_{0}$ is independent of $\tilde{z}$, we use matching condition 4.37) and

$$
\lim _{Z \rightarrow 0} C_{0}=\lim _{\tilde{z} \rightarrow \infty} \tilde{c}_{0}
$$

to note that

$$
c_{0 t}(z)=\left.c_{0 t}\right|_{z \rightarrow \infty}=\left.\tilde{c}_{0 t}\right|_{\tilde{z}=0}=\left.\tilde{c}_{0 t}\right|_{\tilde{z} \rightarrow \infty}=\left.C_{0 t}\right|_{Z=0}=0
$$

since $C_{0}$ is independent of $t$. Thus 4.45 reduces to

$$
\nabla_{x}^{2} c_{1}=0
$$

Integrating 4.51 over $\omega_{h_{0}}(t)$, and using the boundary conditions 4.46 -4.48), the periodicity of $c_{1}$ in $x$, the fact that $c_{0}$ is independent of $x, t$, we find that

$$
\begin{aligned}
0=\iint_{\omega_{h_{0}}(t)} \nabla_{x}^{2} c_{1} \mathrm{~d} x \mathrm{~d} z & =\int_{z=h_{0}}-\beta^{*} c_{0}+\frac{\left(H_{T}+h_{0 t}\right)}{\sqrt{1+h_{0 x}^{2}}} c_{0} \mathrm{~d} s+\left.\int_{x=-\frac{1}{2}}^{\frac{1}{2}} c_{1 z}\right|_{z \rightarrow \infty} \mathrm{d} x \\
& =-\left(\beta^{*} c_{0}+\gamma \beta^{*} c_{0}^{2}\right) \mathcal{L}\left(h_{0}\right)+\left.\int_{x=-\frac{1}{2}}^{\frac{1}{2}} c_{1 z}\right|_{z \rightarrow \infty} \mathrm{d} x
\end{aligned}
$$

where we have defined

$$
\mathcal{L}\left(h_{0}\right)=\int_{z=h_{0}} \mathrm{~d} s
$$

to be the length of the fluid-fluid interface in the cell. We note that matching the fluxes directly between the inner and outer solutions would require that the outer solution is 
a function of the fast time. Since we have already shown that this is not the case, we conclude that there must be an intermediate region between the two in which the fast variations due to the chemistry at the interface will be smoothed out. Before proceeding to consider the intermediate layer, we multiply $(4.47)$ by $\sqrt{1+h_{0 x}^{2}}$ and integrate over $t$ from 0 to $1 / H_{T}$, to find that

$$
1=-\gamma \beta^{*} c_{0} \int_{0}^{1 / H_{T}} \sqrt{1+h_{0 x}^{2}} \mathrm{~d} t,
$$

using the periodicity of $h_{0}$ in $t$. Since $c_{0}$ is independent of $x, h_{0 x}$ must be constant through the cell. For periodicity of $h_{0}$ in $x$, we require that $h_{0 x}=0$, and so we see that the microscale interface is flat to leading order. Thus, 4.55 reduces to

$$
H_{T}=-\gamma \beta^{*} c_{0}
$$

\subsubsection{Intermediate Region}

We now consider the intermediate region. The leading-order problem is:

$$
\nabla_{\xi}^{2} \tilde{c}_{0}=0
$$

in $\omega_{c}(t)$, with, on $\partial \omega_{s}$

$$
\nabla_{\xi} \tilde{c}_{0} \cdot \boldsymbol{n}_{s}=0 .
$$

As in the outer region, this problem, along with the assumption of the spatial periodicity of $\tilde{c}_{0}$ in $\xi$ and $\eta$, gives that $\tilde{c}_{0}$ is independent of $\xi$ and $\eta$. At $\mathcal{O}\left(\epsilon^{1 / 2}\right)$ we have

$$
\nabla_{\xi}^{2} \tilde{c}_{1 / 2}=0
$$

in $\omega_{c}(t)$, with, on $\partial \omega_{s}$,

$$
\left(\nabla_{\xi} \tilde{c}_{1 / 2}+\tilde{c}_{0 \tilde{z}} \boldsymbol{e}_{2}\right) \cdot \boldsymbol{n}_{s}=0
$$

We recall that, from 4.43$), \tilde{c}_{0} \tilde{z}=0$ at $\tilde{z}=0$. We have two options. If $\tilde{c}_{0 \tilde{z}}=0$ for all $\tilde{z}$, then we obtain that $\tilde{c}_{1 / 2}$ is independent of $\xi, \eta$, exactly as for $\tilde{c}_{0}$. If not, (i.e., for some $\tilde{z}, \tilde{c}_{0 \tilde{z}} \neq 0$ ), then we can write $\tilde{c}_{1 / 2}=W \tilde{c}_{0 \tilde{z}}$ with $W$ the solution of cell problem (4.24)4.25 along with periodic boundary conditions. Let us assume for contradiction that we are in this second scenario. With $\tilde{c}_{1 / 2}=W \tilde{c}_{0 \tilde{z}}$, the $\mathcal{O}(\epsilon)$ problem reads

$$
\tilde{c}_{0 t}-H_{T} \tilde{c}_{0 \eta}=\nabla_{\xi}^{2} \tilde{c}_{1}+\left(\nabla_{\xi} \cdot \boldsymbol{e}_{2} \frac{\partial}{\partial \tilde{z}}+\boldsymbol{e}_{2} \frac{\partial}{\partial \tilde{z}} \cdot \nabla_{\xi}\right) \tilde{c}_{1 / 2}+\tilde{c}_{0 \tilde{z} \tilde{z}}
$$

in $\omega_{c}(t)$ with, on $\partial \omega_{s}$,

$$
\left(\nabla_{\xi} \tilde{c}_{1}+\tilde{c}_{1 / 2 \tilde{z}} \boldsymbol{e}_{2}\right) \cdot \boldsymbol{n}_{s}=0
$$

with the usual periodicity of $\tilde{c}_{1}$ in $\xi, \eta$ and $t$. Using the fact that $\tilde{c}_{0}$ is independent of $\xi, \eta$, we integrate 4.61 over $\omega_{c}(t)$ and obtain, by applying the boundary conditions and periodicity and using $\tilde{c}_{1 / 2}=W \tilde{c}_{0 \tilde{z}}$, that

$$
\tilde{c}_{0 t}=D^{*} \tilde{c}_{0} \tilde{z} \tilde{z}
$$

with $D^{*}$ defined as in 4.32. We also have the matching condition 4.49, as well as $\tilde{c}_{0 \tilde{z}}=0$ at $\tilde{z}=0$ from the inner problem. This problem has the unique solution $\tilde{c}_{0}=$ 
$\left.C_{0}\right|_{Z=0}$ for all $\tilde{z}$, as shown in Appendix A, and hence $\tilde{c}_{0 \tilde{z}}=0$ for all $\tilde{z}$, giving the required contradiction.

Since $\tilde{c}_{1 / 2}$ is thus independent of $\xi$ and $\eta$, the $\mathcal{O}(\epsilon)$ problem $4.61-4.62$ reduces to

$$
\nabla_{\xi}^{2} \tilde{c}_{1}=0
$$

in $\omega_{c}(t)$, with, on $\partial \omega_{s}$

$$
\left(\nabla_{\xi} \tilde{c}_{1}+\tilde{c}_{1 / 2 \tilde{z}} \boldsymbol{e}_{2}\right) \cdot \boldsymbol{n}_{s}=0,
$$

which has the solution $\tilde{c}_{1}=W \tilde{c}_{1 / 2 \tilde{z}}$, where $W$ solves the cell problem 4.24)-4.25) with periodicity of $W$ in $\xi, \eta$ and $t$.

At $\mathcal{O}\left(\epsilon^{3 / 2}\right)$

$$
\tilde{c}_{1 / 2 t}-H_{T} \tilde{c}_{1 / 2 \eta}=\nabla_{\xi}^{2} \tilde{c}_{3 / 2}+\left(\nabla_{\xi} \cdot \boldsymbol{e}_{2} \frac{\partial}{\partial \tilde{z}}+\boldsymbol{e}_{2} \frac{\partial}{\partial \tilde{z}} \cdot \nabla_{\xi}\right) \tilde{c}_{1}+\tilde{c}_{1 / 2 \tilde{z} \tilde{z}}
$$

in $\omega_{c}(t)$ (where we have used that $\tilde{c}_{0} \tilde{z}=0$ ) with, on $\partial \omega_{s}$,

$$
\left(\nabla_{\xi} \tilde{c}_{3 / 2}+\tilde{c}_{1 \tilde{z}} \boldsymbol{e}_{2}\right) \cdot \boldsymbol{n}_{s}=0 .
$$

Integrating 4.66) over $\omega_{c}(t)$, using Reynolds Transport Theorem 4.28) for the left-hand side and the divergence theorem for the first two terms on the right, we obtain

$$
\begin{aligned}
\frac{\mathrm{d}}{\mathrm{d} t} \iint_{\omega_{c}} \tilde{c}_{1 / 2} \mathrm{~d} \xi \mathrm{d} \eta & =\int_{\partial \omega_{c}}\left(\nabla_{\xi} \tilde{c}_{3 / 2}+\tilde{c}_{1 \tilde{z}} \boldsymbol{e}_{2}\right) \cdot \boldsymbol{n} \mathrm{d} s+\iint_{\omega_{c}} \boldsymbol{e}_{2} \frac{\partial}{\partial \tilde{z}} \cdot \nabla_{\xi} \tilde{c}_{1}+\left(\tilde{c}_{1 / 2}\right) \tilde{z} \tilde{z} \mathrm{~d} \xi \mathrm{d} \eta \\
& =\mathcal{V} \lambda(t) \tilde{c}_{1 / 2} \tilde{z} \tilde{z},
\end{aligned}
$$

where we have used (4.67) and the periodicity over the cell to evaluate the line integral to zero, and the form of $\tilde{c}_{1}=W\left(\tilde{c}_{1 / 2}\right)_{\tilde{z}}$ and the independence of $\tilde{c}_{1 / 2}$ on $\xi$ and $\eta$ for the final term. Integrating 4.68 over a period of $t$, by the periodicity of $\tilde{c}_{1 / 2}$ in $t$, we find that

$$
0=\int_{t=0}^{1 / H_{T}} \lambda(t) \tilde{c}_{1 / 2 \tilde{z} \tilde{z}} \mathrm{~d} t=\left(\int_{t=0}^{1 / H_{T}} \lambda(t) \tilde{c}_{1 / 2 \tilde{z}} \mathrm{~d} t\right)_{\tilde{z}}
$$

Finally, we integrate 4.69 over $0 \leq \tilde{z} \leq \infty$, and apply the matching conditions

$$
\lim _{Z \rightarrow 0} C_{0 Z}=\lim _{\tilde{z} \rightarrow \infty} \tilde{c}_{1 / 2 \tilde{z}}, \quad \quad \lim _{\tilde{z} \rightarrow 0} \tilde{c}_{1 / 2 \tilde{z}}=\lim _{z \rightarrow \infty}\left(c_{1 z}(z)-\left.z \tilde{c}_{0 \tilde{z} \tilde{z}}\right|_{\tilde{z}=0}\right)
$$

(see $[2]$ ) and 4.53 to find that, on $Z=0$

$$
C_{0 Z}=\left.\frac{H_{T}}{D^{*}} \int_{0}^{1 / H_{T}} \lambda(t) c_{1 z}\right|_{z \rightarrow \infty} \mathrm{d} t=\left(\frac{H_{T}}{D^{*}} \int_{0}^{1 / H_{T}} \lambda(t) \mathcal{L}\left(h_{0}\right) \mathrm{d} t\right)\left(\beta^{*} C_{0}+\gamma \beta^{*} C_{0}^{2}\right) .
$$

The intermediate region allows us to match accurately between the inner and outer problems to give the macroscale boundary condition 4.71). From 4.56) and the matching conditions, we also have

$$
H_{T}=-\left.\gamma \beta^{*} C_{0}\right|_{Z=0} .
$$

We use 4.72 to re-write 4.71 in the same form as for the microscale problem, namely as

$$
H_{T} C_{0}+\mu^{*} C_{0 Z}=\beta^{*} C_{0},
$$


on $Z=0$, where

$$
\mu^{*}=\frac{D^{*}}{H_{T} \int_{0}^{1 / H_{T}} \lambda(t) \mathcal{L}\left(h_{0}\right) \mathrm{d} t},
$$

We note that, although we have a flat interface, $\mathcal{L}\left(h_{0}\right)$ is not everywhere equal to unity, since the solid in the cell can obstruct the interface; the constant $\mu^{*}$ takes into account the solid microstructure.

\subsection{Summary}

We write the system in terms of the original macroscale variables $X$ and $Y$, where the fluid-fluid interface is located at $Y=H$. Dropping the subscript 0 notation, our macroscale model reads:

$$
C_{T}=D^{*} C_{Y Y}
$$

in $Y>H(T)$ with, on $Y=H(T)$

$$
H_{T} C+\mu^{*} C_{Y}=\beta^{*} C, \quad H_{T}=-\gamma \beta^{*} C,
$$

where

$$
D^{*}=H_{T} \int_{0}^{1 / H_{T}} \lambda(t) \mathrm{d} t, \quad \mu^{*}=\frac{D^{*}}{H_{T} \int_{0}^{1 / H_{T}} \lambda(t) \mathcal{L}\left(h_{0}\right) \mathrm{d} t}, \quad \lambda=\frac{1}{\mathcal{V}} \iint_{\omega_{c}}\left(W_{\eta}+1\right) \mathrm{d} \xi \mathrm{d} \eta .
$$

To close the model, equations 4.75 and 4.76 must be solved with appropriate boundary conditions (on the other macroscale boundaries of the domain), and initial conditions for $C$ and $H$.

\section{Comparison of models}

In order to compare the two models directly, we present the re-dimensionalised forms in one spatial dimension.

(1) For the case when the agent coats the solid microstructure (the unsaturated agent case) we have, when $\hat{R}=0$,

$$
\hat{c}_{\hat{t}}=\hat{\mathcal{D}}_{0} \hat{c}_{\hat{y} \hat{y}}
$$

while, when $\hat{R}>0$, we have

$$
\hat{c}_{\hat{t}}=\frac{1}{\hat{\mathcal{V}}(\hat{R})}\left(\hat{\mathcal{D}} \hat{\mathcal{V}}(\hat{R}) \hat{c}_{\hat{y}}\right)_{\hat{y}}-\hat{\nu}(\hat{k}+\hat{\chi} \hat{k} \hat{c}) \hat{c}, \quad \hat{R}_{\hat{t}}=-\hat{\chi} \hat{k} \hat{c}
$$

where $\hat{\mathcal{D}}=\hat{D}(\underline{\underline{D}})_{22}\left(\right.$ and $\left.\hat{\mathcal{D}}_{0}=\hat{D}\left(\underline{\underline{D}}^{0}\right)_{22}\right)$, given by 3.35$), \hat{\nu}=\mathcal{L}(\hat{R}) / \mathcal{V}(\hat{R})$, where $\mathcal{V}$ and $\mathcal{L}$ are given respectively by $(3.30$ and 3.33 .

(2) For the saturated case with the sharp macroscale interface, we have to solve

$$
\hat{c}_{\hat{t}}=\hat{\mathcal{D}}_{0} \hat{c}_{\hat{y} \hat{y}}
$$

with, on $\hat{y}=\hat{h}(\hat{t})$,

$$
\hat{c} \hat{h}_{\hat{t}}+\hat{\mu} \hat{c}_{\hat{y}}=\hat{k} \hat{c}, \quad \hat{h}_{\hat{t}}=-\hat{\chi} \hat{k} \hat{c},
$$


where $\hat{\mu}=\hat{D} \mu^{*}$, where $\mu^{*}$ is given by (4.77).

In both cases, the parameters $\mathcal{L}$ and $\mathcal{V}$ depend on the microscale problem through $\hat{\mathcal{D}}_{0}$ or $\hat{\mathcal{D}}$ (as well as explicitly and through $\hat{\nu}$ in Case 1 and through $\hat{\mu}$ in Case 2). In principle we could solve the microscale problem multiple times and tabulate the solutions to get the functions we need to use to solve the macroscale models.

Both the homogenisation procedures result in the same diffusion equation (and diffusivity) in the region where there is no agent. Otherwise, the two models we have derived are quite different in terms of their structure: in the unsaturated case, the chemistry appears as a reaction term in the main equation, reflecting the fact that the chemical reaction happens throughout the material. On the other hand, in the saturated case, the chemistry only appears at the fluid-fluid interface, as it does in the original pore-scale model. We note that, in the saturated case, our homogenised model is similar to that used in $[3]$ and $[6]$, in which the parameters $\hat{\mathcal{D}}$ and $\hat{\mu}$ do not depend on the microstructure. As in these works, we are free to choose any shape for the porous microstructure and only require that this shape is repeated periodically.

\section{Conclusions and discussion}

In this paper, we have examined the problem of decontamination of a porous medium that has been infiltrated by a hazardous chemical agent using an immiscible cleanser. We focused on the porescale and considered two different scenarios: an unsaturated case where the agent partially fills the porespace and a saturated case where it completely fills the porespace. In each case, we homogenised the porescale model to arrive at equations describing the average concentration of cleanser and motion of agent-cleanser boundaries in which the microscale structure and chemistry has been correctly accounted for but, crucially, smoothed out.

In the unsaturated case, our homogenised model involves a reaction-diffusion equation for the concentration of the cleanser coupled with an ordinary differential equation describing the local thickness of the agent layer. In contrast, in the saturated case (when we have a sharp interface) the cleanser moves by diffusion alone and all the chemistry takes place at a macroscopic interface between contaminated and decontaminated regions. The homogenisation process was straightfoward in the unsaturated case, but required the use of multiple timescales and a boundary layer in order to smooth out short-time variations in the full-porespace case.

For the unsaturated model, we prescribed a circular geometry for the solid inclusion. We note that the homogenisation analysis would proceed in a similar fashion if we were to alter the microstructure so that it was more realistic, either by changing the shape or by having larger unit cells that contain multiple inclusions. We anticipate that, while the functional forms of the macroscale parameters would change, the structure of the resulting model would remain the same. In contrast, for the saturated model we did not require any assumptions on the cell geometry other than periodicity, and so the formulae we derived for the macroscopic parameters are generic and hold for any choice of cell geometry. Thus, the structure of this homogenised model is also unaffected by the specific pore geometry. 
In this paper, we have focused on particular parameter choices that result in interesting and practically relevant distinguished limits of our model. It would be interesting to study other possible distinguished limits in order to fully catalogue the behaviour of our existing porescale model.

Our next objective is to compare and contrast the solutions to our models for realistic parameter values and in the limit where the model in unsaturated case develops a sharp interface. In the saturated case, we have restricted our attention to the case where the macroscale interface is flat. We also intend to carry out the homogenisation process for situations where all variables depend on the lateral coordinate. We anticipate that the structural complexity of the problem will increase, since the gradient of the macroscale interface is likely to affect the shape and position of the microscale interface in each cell problem, which might require averaging along the interface rather than imposing periodicity.

There are many extensions to the underpinning model that could be made to more faithfully approximate the real situation. For example, more complex reactions between the agent and the cleanser could be incorporated, although we anticipate that these will pass through the homogenisation process in an identical manner to that presented here. Also, the assumptions that the agent and cleanser are immiscible, or that the agent is neat, could be relaxed. In either case, we would end up having to incorporate additional mass transfer equations. Finally, we could incorporate liquid flow into the model. This would be a non-trivial extension, because we would need to consider both wetting effects and the possibility of viscous fingering. The latter may be advantageous for decontamination because of the increase in overall reaction associated with the increase in the area of the agent-cleanser interface.

In this paper, we have illustrated how homogenisation theory can be used to derive averaged equations and boundary conditions for decontamination that are computationally much simpler to solve than the original equations on a complicated domain that takes all the microstructure into account, while retaining all the pertinent information from the microstructure. More broadly, our models can be used to help answer scientific questions about the efficiency and speed of decontamination protocols for particular types of spills in a more accurate way, since the fine-scale structure and processes have been systematically taken into account.

\section{Acknowledgements}

We are indebted to many extremely useful discussions with Ross Heatlie-Branson, Jon Chapman, Mohit Dalwadi, and Oliver Whitehead. This publication is based on work partially supported by the EPSRC Centre For Doctoral Training in Industrially Focused Mathematical Modelling (EP/L015803/1) in collaboration with Defra. IMG gratefully acknowledges support from the Royal Society through a University Research Fellowship. 


\section{Appendix A}

With the periodicity of $\tilde{c}_{0}$ in $t$, we now prove that there is a unique solution of the problem 4.63, with boundary conditions

$$
\tilde{c}_{0 \tilde{z}}=0 \text { at } \tilde{z}=0, \quad \text { and } \quad \tilde{c}_{0}=0 \text { at } \tilde{z} \rightarrow \infty .
$$

We define $\phi=\tilde{c}_{0}-\left.C_{0}\right|_{Z=0}$, so that $\phi$ solves

$$
\begin{array}{ll}
\phi_{t}=D^{*} \phi_{\tilde{z} \tilde{z}} & \tilde{z} \in[0, \infty) \\
\phi_{\tilde{z}}=0 & \tilde{z}=0 \\
\phi=0 & \tilde{z} \rightarrow \infty \\
\phi(t, \tilde{z})=\phi\left(t+1 / H_{T}, \tilde{z}\right) & \forall t>0, \tilde{z} .
\end{array}
$$

We let $I=1 / 2 \int_{0}^{\infty} \phi^{2} \mathrm{~d} \tilde{z} \geq 0$, then

$$
\frac{\mathrm{d} I}{\mathrm{~d} t}=\int_{0}^{\infty} \phi \phi_{t} \mathrm{~d} \tilde{z}=-\int_{0}^{\infty} \phi_{\tilde{z}}^{2} \mathrm{~d} \tilde{z}+\left[\phi \phi_{\tilde{z}}\right]_{0}^{\infty}=-\int_{0}^{\infty} \phi_{\tilde{z}}^{2} \mathrm{~d} \tilde{z}+0 \leq 0 .
$$

By periodicity of $\phi, I$ must be periodic in $t$, and so we cannot have that $\mathrm{d} I / \mathrm{d} t$ is negative for any $t$. Therefore $\mathrm{d} I / \mathrm{d} t=0$, and so $\phi_{\tilde{z}}=0$ for all $\tilde{z}$. Hence since $\phi \rightarrow 0$ as $\tilde{z} \rightarrow \infty$, $\phi=0$ everywhere. We conclude that $\tilde{c}_{0}=C_{0}(0)$ for all $\tilde{z}$, and $\tilde{c}_{0 \tilde{z}}=0$ for all $\tilde{z}$. This is the required contradiction to our assumption that $\tilde{c}_{0} \tilde{z}$ is not everywhere zero.

\section{References}

[1] M. Bruna and S. J. Chapman. Diffusion in spatially varying porous media. SIAM J. Appl. Math., 75(4):1648-1674, 2015.

[2] S. J. Chapman. Macroscopic models of superconductivity (PhD. Thesis). 1991.

[3] M. Dalwadi, E. Dubrovina, A. Eisentraeger, A. Lee, J. Maestri, B. Matejczyk, D. O'Kiely, M. Stamper, and S. Thomson. Toxic chemical and their neutralising agents in porous media. Tech. Report, ESGI100, http://www.maths-in-industry.org/miis/671/, 2014.

[4] M. P. Dalwadi, M. Bruna, and I. M. Griffiths. A multiscale method to calculate filter blockage. J. Fluid Mech., 809:264-289, 2016.

[5] M. P. Dalwadi, I. M. Griffiths, and M. Bruna. Understanding how porosity gradients can make a better filter using homogenization theory. Proc. Roy. Soc. A, 471(2182):20150464, 2015.

[6] M. P. Dalwadi, D. O'Kiely, S. J. Thomson, T. S. Khaleque, and C. L. Hall. Mathematical modeling of chemical agent removal by reaction with an immiscible cleanser. SIAM J. Appl. Math., 77(6):1937-1961, 2017.

[7] T. Fatima, N. Arab, E. P. Zemskov, and A. Muntean. Homogenization of a reactiondiffusion system modeling sulfate corrosion of concrete in locally periodic perforated domains. J. Eng. Math., 69(2-3):261-276, 2011.

[8] U. Hornung. Homogenization and porous media, volume 6. Springer Science \& Business Media, 2012.

[9] H. E. Huppert and A. W. Woods. Gravity-driven flows in porous layers. J. Fluid Mech., 292:55-69, 1995.

[10] Y. Liu, Z. Zheng, and H. A. Stone. The influence of capillary effects on the drainage of a viscous gravity current into a deep porous medium. J. Fluid Mech., 817:514-559, 2017.

[11] G. Printsypar, M. Bruna, and I. M. Griffiths. The influence of porous-medium microstructure on filtration. J. Fluid Mech., 861:484-516, 2019. 
[12] D. Pritchard, A. W. Woods, and A. J. Hogg. On the slow draining of a gravity current moving through a layered permeable medium. J. Fluid Mech., 444:23-47, 2001.

[13] P. G. Saffman. On the boundary condition at the surface of a porous medium. Studies in Appl. Math., 50(2):93-101, 1971.

[14] R. Schulz and P. Knabner. Derivation and analysis of an effective model for biofilm growth in evolving porous media. Math. Methods in Appl. Sci., 40(8):2930-2948, 2017.

[15] R. Schulz and P. Knabner. An effective model for biofilm growth made by chemotactical bacteria in evolving porous media. SIAM J. Appl. Math., 77(5):1653-1677, 2017. 